\title{
The Pfaffian Technique: A (2 + 1)-Dimensional Korteweg de Vries Equation
}

\author{
Lixiao Zhai, Junxiao Zhao \\ School of Mathematical Sciences, University of Chinese Academy of Sciences, Beijing, China \\ Email:jxzhao@ucas.ac.cn
}

How to cite this paper: Zhai, L.X. and Zhao, J.X. (2016) The Pfaffian Technique: A $(2+1)$-Dimensional Korteweg de Vries Equation. Journal of Applied Mathematics and Physics, 4, 1930-1935.

http://dx.doi.org/10.4236/jamp.2016.410195

Received: September 13, 2016

Accepted: October 23, 2016

Published: October 27, 2016

Copyright $\odot 2016$ by authors and Scientific Research Publishing Inc. This work is licensed under the Creative Commons Attribution International License (CC BY 4.0).

http://creativecommons.org/licenses/by/4.0/

\section{Abstract}

The $(2+1)$-dimensional Korteweg de Vries $(\mathrm{KdV})$ equation, which was first derived by Boiti et al., has been studied by various distinct methods. It is known that this $(2+$ 1)-dimensional $\mathrm{KdV}$ equation has rich solutions, such as multi-soliton solutions and dromion solutions. In the present article, a unified representation of its $\mathrm{N}$-soliton solution is given by means of pfaffian. We'll show that this $(2+1)$-dimensional $\mathrm{KdV}$ equation is nothing but the Plücker identity when its $\tau$-function is given by pfaffian.

\section{Keywords}

The $(2+1)$-Dimensional Korteweg de Vries Equation, Hirota Bilinear Method, Pfaffian, Plücker Identity

\section{Introduction}

The solitary wave, so-called because it often occurs as a single entity and is localized, was first observed by J. Scott Russell on the Edinburgh-Glasgow Canal in 1834. It is known that many nonlinear evolution equations have soliton solutions, such as the Korteweg de Vries equation, the Sin-Gordon equation, the nonlinear Schrödinger equation, the Kadomtsev-Petviashvili equation, the Davey-Stewartson equation, etc. In order to study the property of nonlinear evolution equations, methods are developed to derive solitary wave solution or soliton solution to nonlinear evolution equations. Some of the most important methods are the inverse scattering transformation (IST) [1] method, the bilinear method [2]-[7], symmetry reduction method [8], the Bäcklund or Darboux transformation method [9] and so on. Having soliton solutions is one of the basic integrable properties of nonlinear evolution equations.

In this paper, we are interested in the general expression of $N$-soliton solution to the 
$(2+1)$-dimensional $\mathrm{KdV}$ equation,

$$
\begin{aligned}
& \frac{\partial u}{\partial t}+3 \frac{\partial(u v)}{\partial x}+\frac{\partial^{3} u}{\partial x^{3}}=0, \\
& \frac{\partial u}{\partial x}-\frac{\partial v}{\partial y}=0,
\end{aligned}
$$

which was first derived by Boiti et al. by using the idea of the weak Lax pair [10]. This system can also be obtained from the inner parameter-dependent symmetry constraint of the KP equation [11]. Recently, the dromion solutions and some exact solutions are studied by Lou and Wazwaz respectively [12]-[14]. While as for the uniformed expression of its $N$-soliton solution is unknown yet.

In this article, we'll study the $N$-soliton solution to the $(2+1)$-dimensional $\mathrm{KdV}$ system (1). A compact form of the $N$-soliton solution to Equation (1) is obtained by means of pfaffian technique, which is given in section 2. Conclusion and further discussions are given in section 3 .

\section{2. $N$-Soliton Solution to the $(2+1)$-Dimensional KdV Equation}

Given a nonlinear evolution equation, if it has 3-soliton solution, then this equation is of great possibility of having $N$-soliton ( $3 \leq N$ ) solution. Pfaffian technique is one of the methods that can help us to determine whether the evolution equation has multisoliton solutions or not. In this section, we first review some properties of pfaffian.

\subsection{Pfaffian}

Pfaffians are antisymmetric functions with respect to its independent variables

$$
\operatorname{pf}(a, b)=-\operatorname{pf}(b, a), \quad \text { for any } a \text { and } b \text {. }
$$

An $n$-th order pfaffian pf $(1,2, \cdots, 2 n)$ can be defined inductively by the expansion rule [3]

$$
\operatorname{pf}(1,2, \cdots, 2 n)=\sum_{j=2}^{2 n}(-1)^{j} \operatorname{pf}(1, j)(2,3, \cdots, \hat{j}, \cdots, 2 n) \text {, }
$$

where $\hat{j}$ denotes the absence of letter $j$. For example, when $n=2$, we have

$$
\operatorname{pf}(1,2,3,4)=\operatorname{pf}(1,2) \operatorname{pf}(3,4)-\operatorname{pf}(1,3) \operatorname{pf}(2,4)+\operatorname{pf}(1,4) \operatorname{pf}(2,3) \text {. }
$$

There are various kinds of pfaffian identities. In this article, we just introduce the so-called Plüker relation for pfaffians [3]

$$
\begin{aligned}
& \operatorname{pf}\left(\alpha_{1}, \alpha_{2}, \alpha_{3}, 1,2, \cdots, 2 n-1\right) \operatorname{pf}(1,2, \cdots, 2 n) \\
& =\operatorname{pf}\left(\alpha_{1}, 1,2, \cdots, 2 n-1\right) \operatorname{pf}\left(\alpha_{2}, \alpha_{3}, 1,2, \cdots, 2 n\right) \\
& \quad-\operatorname{pf}\left(\alpha_{2}, 1,2, \cdots, 2 n-1\right) \operatorname{pf}\left(\alpha_{1}, \alpha_{3}, 1,2, \cdots, 2 n\right) \\
& \quad+\operatorname{pf}\left(\alpha_{3}, 1,2, \cdots, 2 n-1\right) \operatorname{pf}\left(\alpha_{1}, \alpha_{2}, 1,2, \cdots, 2 n\right),
\end{aligned}
$$

which we are going to use. Hereafter, we let $(1,2, \cdots, 2 n)$ denote pfaffian pf $(1,2, \cdots, 2 n)$ for simplicity. 


\section{2. $\mathrm{N}$-Soliton Solutions}

The Hirota form of the $(2+1)$-dimensional KdV system (1) is

$$
D_{y}\left[D_{t}+D_{x}^{3}\right] f \cdot f=0,
$$

which is obtained by the dependent variable transformations

$$
u=2 \frac{\partial^{2} \ln f(x, y, t)}{\partial x \partial y}, \quad v=2 \frac{\partial^{2} \ln f(x, y, t)}{\partial x^{2}} .
$$

Here the Hirota bilinear operator $D_{x}^{m} D_{t}^{n}$ is defined by

$$
\left.D_{x}^{m} D_{t}^{n} a \cdot b \equiv\left(\frac{\partial}{\partial x}-\frac{\partial}{\partial x^{\prime}}\right)^{m}\left(\frac{\partial}{\partial t}-\frac{\partial}{\partial t^{\prime}}\right)^{n} a(x, t) b\left(x^{\prime}, t^{\prime}\right)\right|_{x^{\prime}=x, t^{\prime}=t},
$$

with $n$ and $m$ are arbitrary nonnegative integers.

In [14], the 3 -soliton solution to the $(2+1)$-dimensional $\mathrm{KdV}$ system (3) is obtained

$$
f=1+\sum_{i=1}^{3} \exp \left(\theta_{i}\right)+\sum_{1 \leq i<j \leq 3} c_{i j} \exp \left(\theta_{i}+\theta_{j}\right)+c_{123} \exp \left(\theta_{1}+\theta_{2}+\theta_{3}\right)
$$

where

$$
\begin{gathered}
\theta_{i}=k_{i} x+m_{i} y+q_{i} t+\theta_{i}^{0}, q_{i}=-k_{i}^{3}, k_{i} \neq 0, m_{i} \neq 0 \text { and } \theta_{i}^{0} \text { are constants; } \\
c_{i j}=\frac{\left(p_{i}-p_{j}\right)\left(m_{i}-m_{j}\right)}{\left(p_{i}+p_{j}\right)\left(m_{i}+m_{j}\right)}, \quad c_{123}=c_{12} c_{23} c_{13},
\end{gathered}
$$

via the perturbation method. It claims that the $N$-soliton solutions for $4 \leq N$ can also be obtained by using perturbation method, but the explicit expression of the multisoliton solution is not given.

In this article, we'll study the the multi-soliton solution to Equation (3) using the pfaffian technique [15] [16]. A compact form of the $N$-soliton solution is given in terms of $N$-th order pfaffian.

Proposition 1. If the $\tau$-function fof the $(2+1)$-dimensional $K d V$ system (3) is given by the pfaffian function

$$
f=\left(d_{0}, \beta_{0}, a_{1}, a_{2}, \cdots, a_{N}, b_{1}, b_{2}, \cdots, b_{N}\right),
$$

whose entries, for $j, k=1,2, \cdots, N$, are defined by

$$
\begin{aligned}
& \left(d_{0}, a_{j}\right)=\exp \left(\eta_{j}\right), \quad\left(d_{0}, b_{j}\right)=-1, \quad\left(d_{0}, \beta_{0}\right)=1, \quad\left(a_{j}, a_{k}\right)=a_{j k} \exp \left(\eta_{j}+\eta_{k}\right), \\
& \left(b_{j}, \beta_{0}\right)=1, \quad\left(a_{j}, \beta_{0}\right)=0, \quad\left(b_{j}, b_{k}\right)=b_{j k}, \quad\left(a_{j}, b_{k}\right)=\delta_{j k}, \\
& \text { with } \quad a_{j k}=\frac{p_{j}-p_{k}}{p_{j}+p_{k}}, \quad b_{j k}=\frac{m_{j}-m_{k}}{m_{j}+m_{k}}, \quad \delta_{j k}= \begin{cases}1 & \text { if } j=k \\
0 & \text { if } j \neq k\end{cases} \\
& \eta_{j}=p_{j} x+m_{j} y-p_{j}^{3} t+\eta_{j}^{0}, \quad p_{j} \neq 0, m_{j} \neq 0 \text { and } \eta_{j}^{0} \text { are constants, }
\end{aligned}
$$

then this particular pfaffian function (6) gives an $\mathrm{N}$-soliton solutions to the $(2+1)$-dimensional KdV system (3).

Proof. In the following, we will prove that the pfaffian function (6) satisfies the $(2+$ 1)-dimensional KdV Equation (3). By defining "differential operators" $d_{n} \quad(n=1,2, \cdots)$ 


$$
\left(d_{n}, a_{j}\right)=p_{j}^{n} \exp \left(\eta_{j}\right), \quad\left(d_{n}, b_{j}\right)=\left(d_{n}, d_{m}\right)=\left(d_{n}, \beta_{0}\right)=\left(d_{n}, d_{0}\right)=0,
$$

we obtain the following differential formulae

$$
\begin{aligned}
& \frac{\partial f}{\partial x}=-\left(d_{0}, d_{1}, a_{1}, \cdots, a_{N}, b_{1}, \cdots, b_{N}\right), \frac{\partial^{2} f}{\partial x^{2}}=-\left(d_{0}, d_{2}, a_{1}, \cdots, a_{N}, b_{1}, \cdots, b_{N}\right), \\
& \frac{\partial^{3} f}{\partial x^{3}}=-\left(d_{0}, d_{3}, a_{1}, \cdots, a_{N}, b_{1}, \cdots, b_{N}\right)-\left(d_{0}, d_{1}, d_{2}, \beta_{0}, a_{1}, \cdots, a_{N}, b_{1}, \cdots, b_{N}\right), \\
& \frac{\partial f}{\partial t}=\left(d_{0}, d_{3}, a_{1}, \cdots, a_{N}, b_{1}, \cdots, b_{N}\right)-2\left(d_{0}, d_{1}, d_{2}, \beta_{0}, a_{1}, \cdots, a_{N}, b_{1}, \cdots, b_{N}\right) .
\end{aligned}
$$

In order to find the pfaffian expression for the differential functions with derivative of variable $y$, we need to define another letter $\beta$ [17]

$$
\left(\beta, b_{j}\right)=-m_{j}, \quad\left(\beta, a_{j}\right)=\left(\beta, \beta_{0}\right)=\left(\beta, d_{n}\right)=0 .
$$

Then we have

$$
\begin{aligned}
& \frac{\partial f}{\partial y}=\left(d_{0}, \beta, a_{1}, \cdots, a_{N}, b_{1}, \cdots, b_{N}\right), \\
& \frac{\partial^{2} f}{\partial x \partial y}=\left(d_{0}, d_{1}, \beta, \beta_{0}, a_{1}, \cdots, a_{N}, b_{1}, \cdots, b_{N}\right), \\
& \frac{\partial^{3} f}{\partial x^{2} \partial y}=\left(d_{0}, d_{2}, \beta, \beta_{0}, a_{1}, \cdots, a_{N}, b_{1}, \cdots, b_{N}\right), \\
& \frac{\partial^{4} f}{\partial x^{3} \partial y}=\left(d_{0}, d_{3}, \beta, \beta_{0}, a_{1}, \cdots, a_{N}, b_{1}, \cdots, b_{N}\right)-\left(d_{0}, d_{1}, d_{2}, \beta, a_{1}, \cdots, a_{N}, b_{1}, \cdots, b_{N}\right), \\
& \frac{\partial^{2} f}{\partial y \partial t}=-\left(d_{0}, d_{3}, \beta, \beta_{0}, a_{1}, \cdots, a_{N}, b_{1}, \cdots, b_{N}\right)-2\left(d_{0}, d_{1}, d_{2}, \beta, a_{1}, \cdots, a_{N}, b_{1}, \cdots, b_{N}\right) .
\end{aligned}
$$

Substituting formulae (9) and (11) into the right hand side of Equation (3), we obtain nothing but the Plücker relation for pfaffians (2)

$$
\begin{aligned}
D_{y}\left[D_{t}+D_{x}^{3}\right] f \cdot f= & f\left(\frac{\partial^{2} f}{\partial y \partial t}+\frac{\partial^{4} f}{\partial x^{3} \partial y}\right)-3 \frac{\partial f}{\partial x} \frac{\partial^{3} f}{\partial x^{2} \partial y}+3 \frac{\partial^{2} f}{\partial x \partial y} \frac{\partial^{2} f}{\partial x^{2}}-\frac{\partial f}{\partial y}\left(\frac{\partial f}{\partial t}+\frac{\partial^{3} f}{\partial x^{3}}\right) \\
= & 3\left[\left(d_{1}, \cdots\right)\left(d_{2}, \beta_{3}, \beta_{0}, \cdots\right)-\left(d_{2}, \cdots\right)\left(d_{1}, \beta_{3}, \beta_{0}, \cdots\right)\right. \\
& \left.+\left(\beta_{3}, \cdots\right)\left(d_{1}, d_{2}, \beta_{0}, \cdots\right)-\left(\beta_{0}, \cdots\right)\left(d_{1}, d_{2}, \beta_{3}, \cdots\right)\right] \\
\equiv & 0
\end{aligned}
$$

where $(\cdots)$ denotes $\left(d_{0}, a_{1}, \cdots, a_{N}, b_{1}, \cdots, b_{N}\right)$. Therefore the pfaffian function (6) solves the $(2+1)$-dimensional KdV system (3).

Note that in order to derive the differential formulae of the pfaffian function (6), we have to define another extra letter $\beta$ besides the "differential operators" $d_{n}$. The multi-soliton solution to the nonlinear $(2+1)$-dimensional $\mathrm{KdV}$ system $(1)$ can be obtained by substituting pfaffian function (6) into the dependent variable transformation (4) directly.

\section{Conclusion}

In this article, a compact form of the multi-soliton solution to the $(2+1)$-dimensional 
$\mathrm{KdV}$ system is given via the pfaffian technique. As one can see, the key point of the proof is to derive suitable expressions of the differential formulae of pfaffian $\tau$-function $f$. It is worth pointing out that the method used in this article is different as the one for the proof of the BKP equation, which the differential formulae of the pfaffian $\tau$-function depend only on the "differential operators" $d_{n}$.

\section{Acknowledgements}

We thank the editor and the referee for their comments. This research work is supported by the National Science Foundation of China (Grant Numbers 11271362, 11271266 and 11501510) and a President's Grant from the University of Chinese Academy of Sciences. These supports are greatly appreciated.

\section{References}

[1] Ablowitz, M.J., Kaup, D.J., Newell, A.C. and Segur, H. (1974) The Inverse Scattering Transform-Fourier Analysis for Nonlinear Problems. Studies in Applied Mathematics, 53, 249-315. http://dx.doi.org/10.1002/sapm1974534249

[2] Hirota, R. and Satsuma, J. (1976) N-Soliton Solutions of Model Equations for Shallow Water Waves. Journal of the Physical Society of Japan, 40, 611-612. http://dx.doi.org/10.1143/JPSJ.40.611

[3] Hirota, R. (2004) The Direct Method in Soliton Theory. Cambridge University Press, Cambridge. http://dx.doi.org/10.1017/CBO9780511543043

[4] Hirota, R. (1971) Exact Solutions of the Korteweg-de Vries Equation for Multiple Collisions of Solitons. Physical Review Letters, 27, 1192-1194. http://dx.doi.org/10.1103/PhysRevLett.27.1192

[5] Hereman, W. and Zhuang, W. (1992) Symbolic Computation of Solitons with Macsyma. Comput. Appl. Math. II: Diff. Eq., 2, 287-296.

[6] Sawada, K. and Kotera, T. (1974) A Method for Finding N-Soliton Solutions of the KdV Equation and KdV-Like Equation. Progress of Theoretical Physics, 51, 1355-1367. http://dx.doi.org/10.1143/PTP.51.1355

[7] Matsuno, Y. (1984) Bilinear Transformation Method. Academic Press, New York.

[8] Olver, P.J. (1986) Application of Lie Group to Differential Equation. Springer, New York. http://dx.doi.org/10.1007/978-1-4684-0274-2

[9] Matveev, V.B. and Salle, M.A. (1991) Darboux Transformations and Solitons. Springer, Berlin. http://dx.doi.org/10.1007/978-3-662-00922-2

[10] Boiti, M., Leon, J., Manna, M. and Pempinelli, F. (1986) On the Spectral Transform of Korteweg-de Vries Equation in Two Spatial Dimensions. Inverse Problem, 2, 271-279. http://dx.doi.org/10.1088/0266-5611/2/3/005

[11] Lou, S.Y. and Hu, X.B. (1997) Infinitely Many Lax Pairs and Symmetry Constraints of the KP Equation. Journal of Mathematical Physics, 38, 6401-6427. http://dx.doi.org/10.1063/1.532219

[12] Lou, S.Y. (1995) Generalized Dromion Solutions of the (2+1)-Dimensional KdV Equation. Journal of Physics A: Mathematical and General, 28, 7227-7232. http://dx.doi.org/10.1088/0305-4470/28/24/019

[13] Lou, S.Y. and Ruan, H.Y. (2001) Revisitation of the Localized Excitations of the 2+1 Di- 
mensional KdV Equarion. Journal of Physics A: Mathematical and General, 35, 305-316. http://dx.doi.org/10.1088/0305-4470/34/2/307

[14] Wazwaz, A.M. (2008) Single and Multiple-Soliton Solutions for the (2+1)-Dimensional KdV Equation. Applied Mathematics and Computation, 204, 20-26. http://dx.doi.org/10.1016/j.amc.2008.05.126

[15] Zhao, J.X. and Tam, H.W. (2006) Soliton Solutions of a Coupled Ramani Equation. Applied Mathematics Letters, 19, 307-313. http://dx.doi.org/10.1016/j.aml.2005.01.006

[16] Li, C.X. and Zeng, Y.B. (2007) Soliton Solutions to a Higher Order Ito Equation: Pfaffian Technique. Physics Letters A, 363, 1-4. http://dx.doi.org/10.1016/j.physleta.2006.10.080

[17] Hirota, R., Hu, X.B. and Tang, X.Y. (2003) A Vector Potential KdV Equation and Ito Equation: Soliton Solutions, Bilinear Bäcklund Transformations and Lax Pairs. Journal of Mathematical Analysis and Applications, 288, 326-348.

http://dx.doi.org/10.1016/j.jmaa.2003.08.046

\section{Submit or recommend next manuscript to SCIRP and we will provide best service} for you:

Accepting pre-submission inquiries through Email, Facebook, LinkedIn, Twitter, etc. A wide selection of journals (inclusive of 9 subjects, more than 200 journals)

Providing 24-hour high-quality service

User-friendly online submission system

Fair and swift peer-review system

Efficient typesetting and proofreading procedure

Display of the result of downloads and visits, as well as the number of cited articles

Maximum dissemination of your research work

Submit your manuscript at: http://papersubmission.scirp.org/

Or contact jamp@scirp.org 